\title{
Linguistic validation of the simplified Chinese version of the US National Cancer Institute's patient-reported outcomes version of the common terminology criteria for adverse events (PRO-CTCAE ${ }^{\mathrm{TM}}$ )
}

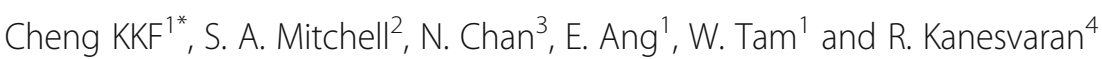

\begin{abstract}
Background: The aim of this study was to translate and linguistically validate the U.S. National Cancer Institute's Patient-Reported Outcomes version of the Common Terminology Criteria for Adverse Events (PRO-CTCAE ${ }^{T M}$ ) into Simplified Chinese for use in Singapore.
\end{abstract}

Methods: All 124 items of the English source PRO-CTCAE item library were translated into Simplified Chinese using internationally established translation procedures. Two rounds of cognitive interviews were conducted with 96 cancer patients undergoing adjuvant treatment to determine if the translations adequately captured the PROCTCAE source concepts, and to evaluate comprehension, clarity and ease of judgement. Interview probes addressed the 78 PRO-CTCAE symptom terms (e.g. fatigue), as well as the attributes (e.g. severity), response choices, and phrasing of 'at its worst'. Items that met the a priori threshold of $\geq 20 \%$ of participants with comprehension difficulties were considered for rephrasing and retesting. Items where $<20 \%$ of the sample experienced comprehension difficulties were also considered for rephrasing if better phrasing options were available.

Results: A majority of PRO-CTCAE-Simplified Chinese items were well comprehended by participants in Round 1. One item posed difficulties in $\geq 20 \%$ and was revised. Two items presented difficulties in $<20 \%$ but were revised as there were preferred alternative phrasings. Twenty-four items presented difficulties in $<10 \%$ of respondents. Of these, eleven items were revised to an alternative preferred phrasing, four items were revised to include synonyms. Revised items were tested in Round 2 and demonstrated satisfactory comprehension.

Conclusions: PRO-CTCAE-Simplified Chinese has been successfully developed and linguistically validated in a sample of cancer patients residing in Singapore.

Keywords: PRO-CTCAE, Patient-reported outcomes, Symptomatic adverse events, Linguistic validation

\footnotetext{
* Correspondence: nurckfk@nus.edu.sg

'Alice Lee Centre for Nursing Studies, Yong Loo Lin School of Medicine, National University of Singapore, Singapore, Singapore

Full list of author information is available at the end of the article
}

(c) The Author(s). 2020 Open Access This article is licensed under a Creative Commons Attribution 4.0 International License, which permits use, sharing, adaptation, distribution and reproduction in any medium or format, as long as you give appropriate credit to the original author(s) and the source, provide a link to the Creative Commons licence, and indicate if changes were made. The images or other third party material in this article are included in the article's Creative Commons licence, unless indicated otherwise in a credit line to the material. If material is not included in the article's Creative Commons licence and your intended use is not permitted by statutory regulation or exceeds the permitted use, you will need to obtain permission directly from the copyright holder. To view a copy of this licence, visit http://creativecommons.org/licenses/by/4.0/. The Creative Commons Public Domain Dedication waiver (http://creativecommons.org/publicdomain/zero/1.0/) applies to the data made available in this article, unless otherwise stated in a credit line to the data. 


\section{Background}

There is increasing recognition in both clinical research and in care delivery of the importance of using patientreported outcomes (PROs) to directly capture the patient's perspective of their treatment experiences, including treatment-related toxicities. A recent review highlighted that the measurement of PROs could provide considerable added value towards the interpretation of study-related outcomes by both trialists and clinicians, and may also strengthen the quality of clinician-patient interactions, and assist regulatory agencies with decision-making [1].

The Patient-Reported Version of the Common Terminology Criteria for Adverse Events (PRO-CTCAE ${ }^{\mathrm{mm}}$ ), was developed by the US National Cancer Institute (NCI), to be used in conjunction with the Common Terminology Criteria for Adverse Events (CTCAE) to facilitate patient self-reporting of symptomatic adverse effects. PROCTCAE is designed to supplement clinician-based CTCAE grading, leading to improved characterization and reporting of cancer treatment toxicities [2-4]. CTCAE is the standard for grading and reporting toxicities in cancer clinical trials, and is used to guide treatment decisionmaking and inform the labelling of oncology products [4, 5]. The most recent version of the clinician-based CTCAE, version 5 , is a compendium of 790 discrete adverse events each of which is defined and graded using an ordinal severity scale [3]. Of the 790 adverse events listed in the CTCAE, 78 symptomatic adverse events that are amenable to patient self-reporting (e.g. pain, fatigue) were selected for inclusion in PRO-CTCAE $[2,6]$.

The PRO-CTCAE item library is comprised of 124 items reflecting 78 symptomatic toxicities. PRO-CTCAE items evaluate these symptomatic toxicities with respect to the attributes of frequency, severity, interference with usual or daily activities, presence/absence or amount. PRO-CTCAE items direct the respondent to report the symptomatic adverse event at its worst during the past 7 days. Each PRO-CTCAE item includes a plain language term describing the symptomatic adverse event, and one or more of the attributes that align with the CTCAE grading criteria for that adverse event [2-4]. Iterative cognitive debriefing interviews conducted with 127 participants with a diverse range of cancer diagnoses and lower levels of educational attainment provide evidence that PRO-CTCAE, including the terms, attributes, response options, and recall period, is well understood and meaningful to patients receiving treatment for cancer, thus supporting the content validity of this instrument [4]. A previous validation analysis of the PRO-CTCAE involving 940 participants undergoing cancer treatment demonstrated favourable test-retest reliability, construct validity, and responsiveness [3]. The original English PRO-CTCAE has been translated and linguistically validated in more than 20 languages [6-9]. There is a need for development of a Simplified Chinese-language version of PRO-CTCAE to make the PRO-CTCAE item library accessible to a larger number of patients with cancer. This is important since Chinese-speaking populations constitute approximately $16 \%$ of the global population [10]. Quality translation of the original language, and linguistically validation to ensure conceptual equivalence with the original English source and cultural appropriateness, comprehensibility, clarity and ease of response of the translated PRO-CTCAE-Simplified Chinese items are particularly important. Availability of such a linguistically validated Simplified Chinese version of PRO-CTCAE facilitates continued adoption of this measure in cancer clinical trials. In addition, availability of a Simplified Chinese version of PRO-CTCAE would support international cancer research efforts. It would also increase the availability of internationally comparable data of toxicity information from patients receiving cancer treatments, so as to help identify effective and tolerable cancer treatment regimens and guide provision of targeted supportive care to improve tolerability. Therefore, the aim of this study was to develop PRO-CTCAE in the Simplified Chinese language, and to linguistically validate the translation to ensure conceptual equivalence with the PRO-CTCAE English source, and confirm a high level of comprehension, clarity, and ease of response.

\section{Methods}

PRO-CTCAE-Simplified Chinese was developed, tested and refined using internationally established translation procedures proposed by Wild et al. (2005) [11] and the World Health Organization (2016) [12]. The process describes two stages (1): forward and backward translation and (2) linguistic validation using cognitive debriefing interviews.

\section{Forward and backward translation}

Translation procedures were conducted by two translators. One was a bilingual Singapore-residing speaker who was fluent in Mandarin. The other translator was a trilingual Hong Kong Special Administrative Region China-residing linguist. Both translators were wellversed in Mandarin/ written Chinese, Cantonese and English, and had substantial prior experience translating between English and Simplified Chinese in health care contexts. Each translator performed independent forward translations of the original English language version of PRO-CTCAE into Simplified Chinese. Our goal in selecting translators residing in different Chinese speaking countries was to ensure that item phrasing be well-comprehended and culturally relevant to geographically diverse Chinese-speaking populations. The forward translations were then reconciled and harmonized into one Simplified Chinese version by a bilingual 
investigator in Singapore and a native Chinese-speaking healthcare professional from China. Next, a bilingual healthcare professional and a second bilingual person from Singapore who had not seen the original English version independently back-translated the reconciled Simplified Chinese translation into English. The backward translations were compared with the original English PRO-CTCAE to identify discrepancies. The initial reconciled Simplified Chinese translation was then further refined in an iterative fashion by two bilingual investigators in Singapore, a native Chinese-speaking healthcare professional from China, and a native Chinese-speaking representative from the US NCI. This resulted in a Simplified Chinese translation of PRO-CTCAE with semantic and conceptual equivalence to the original English PROCTCAE and strong suitability for use with Chinesespeakers in diverse regions. This translation was then advanced for linguistic validation in a sample of patients undergoing cancer treatment.

\section{Cognitive debriefing interviews: materials and methods}

The PRO-CTCAE-Simplified Chinese was evaluated through a series of cognitive debriefing interviews with patients undergoing adjuvant treatment. The debriefing interviews were semi-structured and included questions focused on comprehensibility, clarity, ease of judgment, and ease of response. This process aimed to ensure the cultural appropriateness, comprehensibility, clarity and ease of response of the translated PRO-CTCAE-Simplified Chinese items including the symptom terms, item stems, recall period, attributes (i.e. frequency, severity, and interference with usual or daily activities), and response options $[4,13-16]$. The study was conducted at two national cancer centers in Singapore following approval from the respective Institutional Review Boards. Individuals were invited to participate if they were native Chinese speakers who were able to read Simplified Chinese, age 21 years or older, and newly diagnosed with colorectal or breast cancer and undergoing adjuvant chemotherapy. We purposively sampled participants with two common cancers, colorectal or breast cancer, to optimize the pace of study accrual and to assure a balanced representation of women and men. In Singapore, colorectal cancer was the most common cancer diagnosed in men, while breast and colorectal cancers are the two most common cancers in women [17]. Clinical staff at study sites screened potential participants. Eligible participants who expressed an interest in participation were referred by their clinician to research assistants who provided a detailed explanation of the study purpose and data collection procedures, and obtained their consent. This study was conducted in accordance with the Declaration of Helsinki; all participants provided written informed consent at enrollment.
We planned for at least two rounds of interviews in this validation study. We used a process of iterative refinement of items and continued cognitive interviewing until all the PRO-CTCAE-Simplified Chinese items demonstrated good comprehension [6]. Two native Chinese-speaking, bilingual trained interviewers from China and Singapore conducted the cognitive debriefing interviews. In Singapore, more than $70 \%$ of the residential population is ethnically Chinese. Although English is the official language in Singapore, Mandarin Chinese is commonly spoken in daily life among ethnic Chinese populations, and Simplified Chinese characters are the standard character set of the Chinese written language. Mandarin Chinese and Simplified Chinese characters are also the most widely spoken and written languages, both in China and in other Chinese-speaking communities around the world.

In this study, participants completed a four-item measure of acculturation that reflects language preferences (in speech, writing, thought, and social and vocational contexts); lower scores indicate a lower level of English language acculturation [18]. The participants then completed all items in the PRO-CTCAE-Simplified Chinese item library (124 items for females; 121 items for males). Interviewers did not aid study participants in responding to the PRO-CTCAE-Simplified Chinese items. Participants indicated those PRO-CTCAE items they found difficult to understand or difficult to answer. Interviewers also took field notes to record behavioural indicators of possible non-comprehension such as hesitation with responses, changing of responses, or participants' facial expressions or body language that might suggest uncertainty and thus lack of comprehension of the symptom items. After the participant completed the PRO-CTCAE items, the interviewer conducted cognitive debriefing interviews in Mandarin Chinese. Verbal probing was used to elicit the participants' comprehension of the symptom terms (e.g. what does the word 'fatigue' mean to you?), and the attributes (e.g. what does the phrase 'severity at its worst' mean in this question?). In addition, the probes elicited the participants' cognitive processes regarding the distinctions among frequency, severity, and inference, and the recall period, as well as their judgment processes in selecting a response choice $[15,19]$. Probing also focused on those items where a participant had appeared to be hesitant or uncertain in selecting a response, and items the participant had marked as difficult to understand or difficult to answer. If a participant did not indicate that any items as difficult to understand or difficult to answer, the interviewers probed on PRO-CTCAE items for loose or watery stools, shortness of breath, problems with memory, decreased appetite, insomnia, increased passing of gas, flashing lights in front of your eyes, watery eyes, and 
wheezing, as these were symptom terms where challenges arose in the translation process. All interviews were scheduled to coincide with participants' clinical visits; interviews were audio-recorded and conducted in a private room at the study site.

\section{Analysis}

The audio-recorded interviews were transcribed verbatim, and the transcript was verified by another research staff member. Interview data pertaining to symptom terms and attributes were summarized and analysed across participants. Linguistic and cultural themes related to comprehension, cultural appropriateness and relevance, and cognitive processes were categorized, and subsequently analysed to determine semantic and conceptual equivalence with the original English PROCTCAE phrasings. In addition, the proportions of participants who experienced comprehension difficulties with respect to the symptom terms, and the attributes (e.g. frequency, severity, interference) were tabulated. Generally, items indicated as difficult to understand or difficult to answer by $\geq 20 \%$ of participants during Round 1 of the cognitive debriefing interviews were reviewed by the study team and considered for revision and retesting in Round 2. Items experienced as difficult by less than $20 \%$ of the sample were also reviewed, and if better phrasing options were available, revisions were made and retested in Round 2. All decisions regarding revisions considered the interview data, behavioural indicators of possible non-comprehension from the field notes, the characteristics of the participants (e.g. education level), and the availability of suitable alterative phrasings [4].

\section{Results}

During the period of September 2012-December 2015 (except October 2013-March 2014), 81 participants were enrolled in this study, completed the initial version of the PRO-CTCAE-Simplified Chinese, and participated in the first round of cognitive debriefing interviews. From May to December 2016, 15 additional participants enrolled and participated in a second round of cognitive debriefing to evaluate items that were revised after Round 1 . The socio-demographic and clinical characteristics of the interview samples in both rounds were comparable. In the pooled sample, slightly more than $60 \%$ of the participants were aged 50-64 years, and more than $80 \%$ were female. Approximately one third of participants had stage 1 or II disease: a majority of the sample had received treatment with surgery and chemotherapy. Almost one-third (30.2\%) had completed only primary education (approximately equivalent to 6th Grade or less). The sample also had a low level of English acculturation, with more than $80 \%$ reporting that they were most comfortable speaking Chinese, and preferred to speak Chinese with friends, to think in Chinese, and to speak Chinese at home (Table 1). The mean duration of the interviews was $15 \mathrm{~min}$ with a range of 6 to $36 \mathrm{~min}$.

Table 2 summarizes the proportions of participants who indicated that an item was difficult to understand or difficult to answer, together with the items that were rephrased after Round 1, and the final decisions about item phrasing that were made based on the data from Round 2. The symptom term 'Stretch marks' was considered confusing, hence affecting ease of response by $22.2 \%$ of the participants in Round 1. Interview data and field notes revealed that participants were uncertain if the symptom term of 'Stretch marks', referred only to stretch marks that related to pregnancy. This symptom term was revised by adding a Chinese word of 'skin' before the phrasing of 'lines on the body' and was retested in Round 2. There were no further difficulties with this revised item when tested in 15 participants.

PRO-CTCAE-Simplified Chinese items reflecting four PRO-CTCAE symptom terms (bed sores, difficult getting or keeping an erection, ejaculation problems, increased passing of gas) were identified as difficult to comprehend by $10-20 \%$ of respondents. For 'Bed sores' and 'Increased passing of gas', alternative phrasings were proposed by the study team and these items were revised and retested with 15 participants in Round 2. There were no further difficulties with these revised symptom terms in Round 2 of the cognitive debriefing interviews. Since plain language alternative phrasings for 'Difficulty getting or keeping an erection' and 'Ejaculation problem' were not available, they remained unchanged. None of the 15 participants in Round 2 endorsed comprehension difficulties with these symptom terms.

Fewer than $10 \%$ of respondents found that symptom terms addressing flashing lights in front of your eyes, numbness or tingling in your hands or feet, wheezing, hot flashes, general pain, nausea, vaginal dryness, sad or unhappy feelings, feeling that nothing could cheer you up, pain during vaginal sex, pounding or racing heartbeat, bloating, abdominal pain, frequent urination, and urinary urgency were difficult to comprehend or difficult to answer. As described below and in Table 2, alternative phrasings were proposed by either interviewers and/or respondents, and items were revised and retested with 15 participants in Round 2. There were no further difficulties with those revised symptom terms in Round 2 of the cognitive debriefing interviews.

For example, interview data and field notes from Round 1 of the cognitive debriefing interviews revealed that some participants were uncertain about the symptom terms of 'Hot flashes' (e.g. feeling hot, fever, etc.) and the type/ location of general pain (e.g. head, back, etc.), and recommended that an elaboration be included in parentheses. Therefore, elaborations (feeling of 
Table 1 Sample characteristics

\begin{tabular}{|c|c|c|c|}
\hline Characteristics & Pooled sample Rounds $1 \& 2(N=96)$ & Round $1(n=81)$ & Round $2(n=15)$ \\
\hline Age in years (mean $\pm S D$ ) & $55.16 \pm 8.7$ & $55.05 \pm 8.7$ & $55.87 \pm 9.8$ \\
\hline$<50$ & $23(24.0)$ & $20(24.7)$ & $3(20)$ \\
\hline $50-64$ & $61(63.5)$ & $52(64.2)$ & $9(60)$ \\
\hline$\geq 65$ & $12(12.5)$ & $9(11.1)$ & $3(20)$ \\
\hline \multicolumn{4}{|l|}{ Gender } \\
\hline Female & $80(83.3)$ & $68(84)$ & $12(80)$ \\
\hline \multicolumn{4}{|l|}{ Education level } \\
\hline No formal education & $2(2.1)$ & $2(2.5)$ & 0 \\
\hline Primary & $29(30.2)$ & $26(32.1)$ & $3(20)$ \\
\hline Secondary & $54(56.3)$ & $44(54.3)$ & $10(66.7)$ \\
\hline Junior college/diploma & $8(8.3)$ & $6(7.4)$ & $2(13.3)$ \\
\hline Undergraduate to postgraduate & $3(3.1)$ & $3(3.7)$ & 0 \\
\hline Country of birth & $(n=94)$ & $(n=79)$ & \\
\hline China & $8(8.5)$ & $6(7.6)$ & $2(13.3)$ \\
\hline Hong Kong & $1(1.1)$ & $1(1.3)$ & 0 \\
\hline Malaysia & $16(17.0)$ & $15(19)$ & $1(6.7)$ \\
\hline Singapore & $69(73.4)$ & $57(72.2)$ & $12(80)$ \\
\hline \multicolumn{4}{|l|}{ Language acculturation } \\
\hline 75-100\% feel most comfortable speaking Chinese & $79(82.3)$ & $65(80.2)$ & $14(93.3)$ \\
\hline $75-100 \%$ prefer to speak Chinese with friends & $77(80.2)$ & $64(79)$ & $13(86.7)$ \\
\hline 75-100\% think in Chinese & $82(85.4)$ & $69(85.2)$ & $13(86.7)$ \\
\hline 75-100\% speak Chinese at home & $79(82.3)$ & $67(82.7)$ & $12(80)$ \\
\hline \multicolumn{4}{|l|}{ Chinese-speaking region or dialect } \\
\hline Fujian & 39 (40.6) & $34(42.0)$ & $5(33.3)$ \\
\hline Chaoshan & $22(22.9)$ & $17(21.0)$ & $5(33.3)$ \\
\hline Fujian \& Chaoshan & $3(3.1)$ & $3(3.7)$ & 0 \\
\hline Southern region of Guangdong \& Hong Kong & $10(10.4)$ & $8(9.9)$ & $2(13.3)$ \\
\hline North-eastern region of Guangdong & $5(5.2)$ & $4(4.9)$ & $1(6.7)$ \\
\hline Hainan & $4(4.2)$ & $3(3.7)$ & $1(6.7)$ \\
\hline Others & $6(6.3)$ & $5(6.2)$ & $1(6.7)$ \\
\hline Missing & $7(7.3)$ & 7 (8.6) & $0(0)$ \\
\hline \multicolumn{4}{|l|}{ Type of cancer } \\
\hline Breast & $72(75.0)$ & $60(74.1)$ & $12(80)$ \\
\hline Colorectal & $24(25.0)$ & $21(25.9)$ & $3(20)$ \\
\hline \multicolumn{4}{|l|}{ Stage of cancer } \\
\hline$|-| \mid$ & $56(58.3)$ & $48(59.3)$ & $8(53.3)$ \\
\hline III & $34(35.4)$ & $28(34.6)$ & $6(40.0)$ \\
\hline issing & $6(6.3)$ & $5(6.2)$ & $1(6.7)$ \\
\hline Type of cancer therapy & & & $(n=14)$ \\
\hline Chemotherapy & $7(7.4)$ & $3(3.7)$ & $4(28.6)$ \\
\hline Surgery + chemotherapy & $80(84.2)$ & $70(86.4)$ & $10(71.4)$ \\
\hline Surgery + chemo-radiotherapy & $8(8.4)$ & $8(9.9)$ & 0 \\
\hline
\end{tabular}




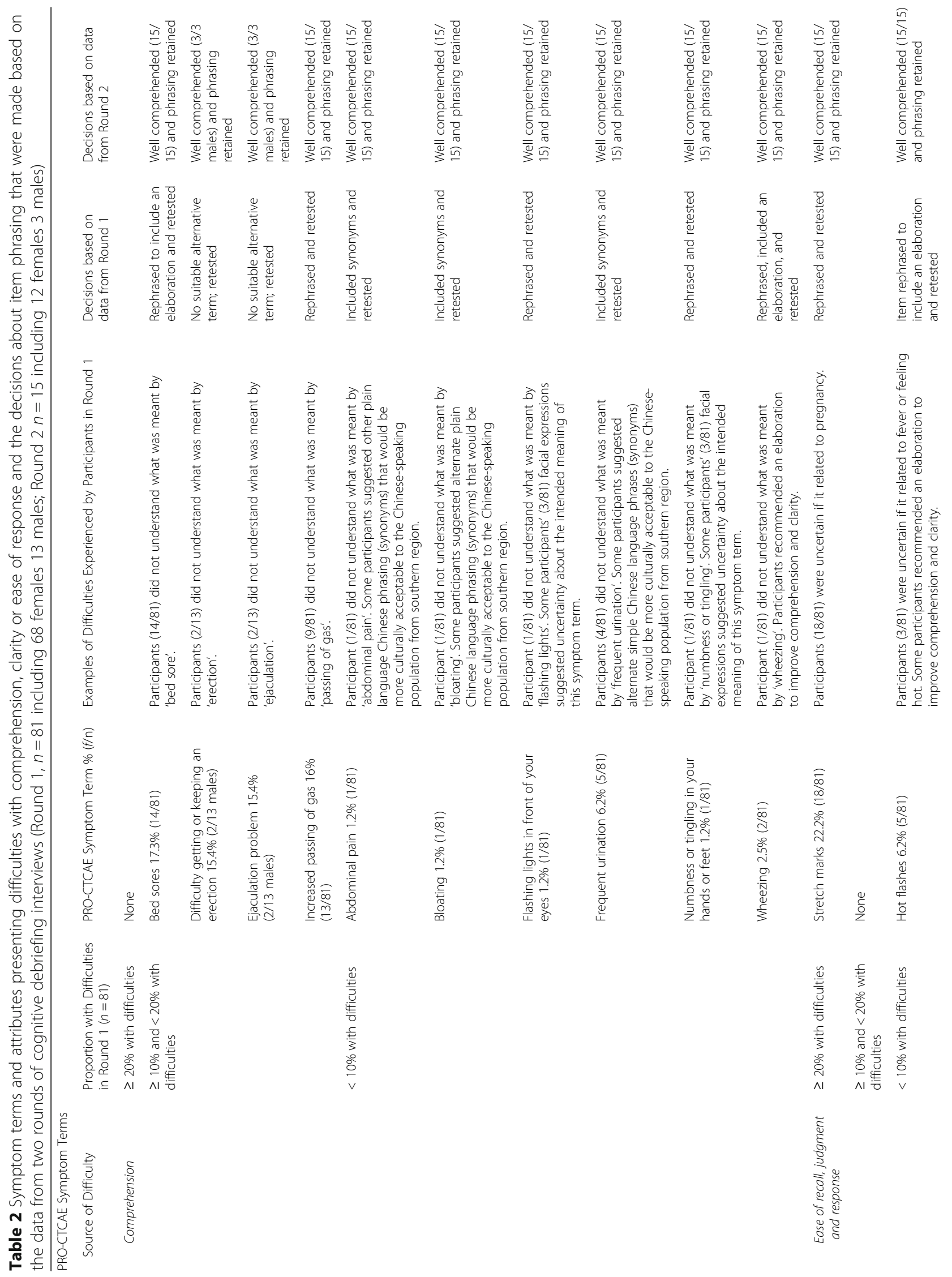




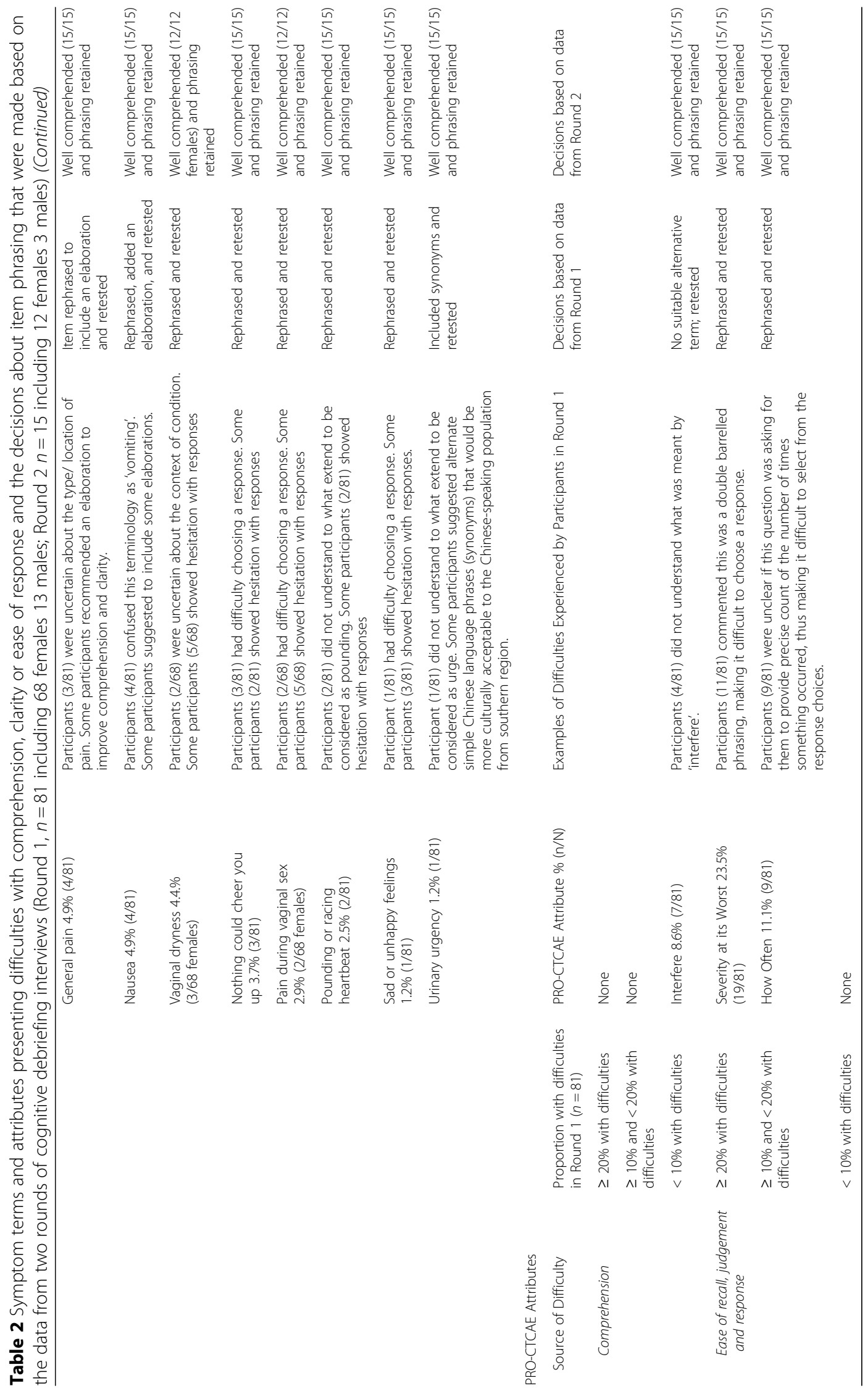


intense heat that may be accompanied with sweating and rapid heartbeat for 'Hot flashes'; experienced in any parts of the body for 'General pain') were added to these two symptom terms, resulting in improved clarity and comprehension. A few participants in Round 1 of the cognitive debriefing interviews suggested synonyms for abdominal pain, bloating, frequent urination, and urinary urgency that they believed would be more culturally acceptable and better comprehended by Chinese-speaking respondents from the southern region. Therefore, the study team decided to incorporate synonyms into items to strengthen the comprehension and cultural acceptability of these items for a diverse range of Chinese speakers. The 15 participants in Round 2, including two from the southern region of Guangdong, indicated that the inclusion of these synonyms improve comprehension and clarity.

Data from Round 1 revealed that the phrasing of the PRO-CTCAE attributes for severity, frequency, and interference was considered difficult to comprehend or difficult to judge by 19 (23.4\%), nine (11.1\%), and seven (8.6\%) out of 81 participants, respectively. Some participants commented that the expression of 'severity at its worst' in Chinese seemed to contain a double-negative, and that as a result it was difficult to select a response option. This element of the PRO-CTCAE translation was reviewed by the study team and was revised to avoid this unintended double-negative phrasing, and to improve the comprehension and clarity of 'severity at its worst' for Chinese speakers. There were no further difficulties when the revised phrasing of 'severity at its worst' was retested in Round 2 with 15 participants. Participants also commented that the phrasing of the frequency question 'how often' was interpreted as requesting a specific count of the number of times something occurred in the past 7 days. As such, respondents felt that the response choices offered (never, occasionally, sometimes, etc.) did not seem well matched to the question phrasing, making it difficult for them to select a response. Better alternative phrasing was available for these frequency items, and there were no further difficulties when the revised phrasing was retested with 15 participants in Round 2. Since better alternative phrasing was not available for the attribute of 'interfere', it remained unchanged.

No patterns in reported comprehension difficulties were observed in subgroups based on educational level, gender or Chinese-speaking geographical region. No participant in Round 1 or Round 2 reported difficulty with the phrasings chosen for the 7-day recall period or the response options. Furthermore, none of the 15 participants in Round 2 reported difficulties in understanding any of the symptom terms including the modified symptom terms and attributes, and thus acceptable comprehension of the finalised version of the PRO-CTCAESimplified Chinese was confirmed.

\section{Discussion}

Sireci (1998) has argued that demonstration of content validity is a fundamental requirement of all assessment instruments [20]. In this study, a cognitive debriefing interview approach [14-16] to content validation provides evidence that the finalized PRO-CTCAE-Simplified Chinese was clearly understood by patients with breast or colorectal cancer from diverse Chinese-speaking regions and with various levels of educational attainment. Our translation and linguistic validation team was comprised of Chinese health care professionals from Singapore, China, and Hong Kong SAR, China who were well-versed in spoken/ written Chinese and English, thereby ensuring that PRO-CTCAE-Simplified Chinese items were culturally relevant and acceptable for use across different Chinese-speaking populations. Our approach to translation and linguistic validation including independent forward and back translations, reconciliation and harmonization, and iterative cognitive debriefing interviews to strengthen and establish conceptual equivalence with PRO-CTCAE English source. Indeed, the inclusion of cognitive debriefing interviews in the process of linguistic validation is crucial to ensure crosscultural content validity of a translated and adapted patient-reported outcome measure [21].

Caveats when interpreting the results of our study include that our sample was comprised of patients with breast or colorectal cancer, and that there were only 16 male participants (13 interviews in Round 1, and 3 interviews in Round 2) in the pooled sample. At the same time, our large sample size and enrichment for lower educational attainment and lower English language acculturations strengthens confidence in the generalizability of our results. Literature suggests that cognitive interviewing studies require approximately 5-15 interviews per item [14].

With our large sample size, we were able to ensure that at least 13 participants or more were debriefed about each of the 124 PRO-CTCAE items. Although among our 13 male respondents in Round 1, there were a few difficulties with comprehension of male-specific symptom terms, there were no other observed patterns by gender with respect to the comprehension, clarity or ease of response of the gender non-specific PROCTCAE symptom terms.

Of note, we found it challenging to develop item phrasings for the PRO-CTCAE question stems reflecting the attributes of frequency and 'severity at its worst'. As the English and Chinese language differ considerably in terms of the grammar and syntax for phrasing questions, phrasing with respect to frequency and severity in the initial PRO-CTCAE-Simplified Chinese version aimed to achieve conceptual equivalence with the English PROCTCAE. Thus, during forward translation, the translators employed phrasing that corresponded closely to the 
English language version. The back translation also reflected conceptual equivalence to the English PROCTCAE source material. Nevertheless, 23.4 and $11.1 \%$ of the participants in Round 1 found the chosen phrasings for the PRO-CTCAE attributes 'severity at its worst' and 'how often', respectively, challenging to interpret in light of the response options. Specifically, the participants understood the expression of 'severity at its worst' in Chinese as a double-negative and interpreted questions asking 'how often' as requesting a specific count of the number of times they experienced a symptom. The study team subsequently revised the Chinese language phrasings of these attributes to achieve improved comprehension and ease of response by Chinese-speaking participants, while also maintaining conceptual equivalence with the English PRO-CTCAE. No further difficulties were reported when the revised attributes of 'severity at its worst' and 'how often' were retested with all of the 15 participants in Round 2.

In this study, phrasings used for PRO-CTCAESimplified Chinese symptom terms were generally wellunderstood, and meaningful to diverse respondents, Overall, our data did not reveal any distinct patterns of difficulty by geographical region or level of educational attainment. At the same time, the interpretation by Chinese speakers of the phrasing chosen for a few specific PRO-CTCAE terms (e.g. abdominal pain, bloating, frequent urination, and urinary urgency) did vary based on geographic region. Accordingly, we added synonyms alongside the original translated phrasings to enhance the culturally acceptability of these symptom terms for diverse Chinese-speaking populations. The participants in Round 2 appreciated the addition of these synonyms, and indicated that their inclusion strengthened comprehension and clarity. The cognitive debriefing interviews conducted in Rounds 1 and 2 further suggested that the recall period, response options, and general instructions were understandable and comprehensible.

Our study results should be interpreted in light of a few limitations. First, our sample may have been biased toward inclusion of participants with preserved performance status since completion of the PRO-CTCAESimplified Chinese survey and the cognitive debriefing interview required that participants have the necessary stamina to complete these study-related procedures. Second, all study participants were residents of Singapore. While we believe that the clinical, demographic, and geographical diversity of our sample support our conclusions about content validity, additional testing to confirm the comprehensibility and cultural acceptability of PRO-CTCAE-Simplified Chinese in other countries is warranted. Third, while our sample was quite large and diverse with respect to geographic region, age and educational attainment, we only sampled patients with breast and colorectal cancer, and the sample was predominantly female. Although we did not observe differences between men and women in the comprehension of gender non-specific symptom terms, the underrepresentation of males in our sample limits our ability to conclude that PRO-CTCAE-Simplified Chinese is well-comprehended by male respondents. Future studies should evaluate PROCTCAE-Simplified Chinese with respondents being treated for a broader range of tumor types, and in samples with more balanced gender distribution. At the same time, confidence in the generalizability of our study findings is strengthened by the rigorous process of translation and cognitive interviewing, and the large sample of geographically diverse group of respondents with low English acculturation.

\section{Conclusion}

Our results support the content validity and acceptability of PRO-CTCAE-Simplified Chinese, and suggest that this translation can be used to capture symptomatic toxicities of cancer treatment in Chinese speakers. Replication and extension of these findings with Chinese speakers residing in other countries is ongoing, and will provide additional evidence to support the comprehensibility and cultural acceptability of PRO-CTCAE-Simplified Chinese in male respondents and for those respondents being treated for a broader range of tumor types. Additional studies to quantitatively examine the reliability and construct validity of PRO-CTCAE-Simplified Chinese are also warranted.

Going forward, PRO-CTCAE-Simplified Chinese provides a common platform by which trialists can capture treatment-related side effects by patient self-report from Chinese speakers. Such information can be used to identify effective and tolerable cancer treatment regimens, to improve shared decision-making between clinicians and patients, and to guide provision of targeted supportive care to improve tolerability.

\section{Abbreviations}

CTCAE: Common Terminology Criteria for Adverse Events; NCl: National Cancer Institute; PROs: Patient-reported outcomes; PRO-CTCAE: PatientReported Outcomes version of the Common Terminology Criteria for Adverse Events

\section{Acknowledgements \\ This study was supported by a Bridging Grant of the National University Health System, Singapore. We thank clinical staff for their assistance in screening/recruitment and Ms. Ethel Lim, Ms. Ge Lixia, and Ms. Parames Khalechelvam for their assistance in recruitment and data collection. We also wish to express our gratitude to the patients for agreeing to participate in this study.}

\section{Authors' contributions}

CKKF designed the work, supported the site work, performed data analyses and interpretation of data, and drafted and finalised the manuscript. MSA contributed to the conception of the work, interpretation of data, and critically reviewed the manuscript for important intellectual content. $\mathrm{CN}$ and AE supported the site work. TW contributed to data analyses. KR supported 
the site work and critically reviewed the manuscript for important intellectual content. All authors approved the manuscript.

\section{Funding}

This work was partially supported by the National University Health System Bridging Funds 02/FY13, Singapore. The funder had no influence on study design, data collection and analyses, or interpretation of data, or writing of the manuscript.

\section{Availability of data and materials}

The data and materials are available from the corresponding author on reasonable request.

\section{Ethics approval and consent to participate}

This study was conducted in accordance with the Declaration of Helsinki and the ethical standards of the institutional review board in Singapore. The study was approved by the Domain Specific Review Board of National Health Group Cluster in Singapore (Study Reference Number: 2015/00039). All participants provided written informed consent at enrolment.

\section{Consent for publication}

Not applicable.

\section{Competing interests}

The authors declare that they have no competing interests.

\section{Author details}

${ }^{1}$ Alice Lee Centre for Nursing Studies, Yong Loo Lin School of Medicine, National University of Singapore, Singapore, Singapore. ${ }^{2}$ Division of Cancer Control and Population Sciences, National Cancer Institute, Rockville, USA. ${ }^{3}$ Division of Palliative Care, National University Cancer Institute, Singapore, Singapore. ${ }^{4}$ Division of Medical Oncology, National Cancer Centre Singapore, Singapore, Singapore.

Received: 22 March 2020 Accepted: 12 November 2020 Published online: 26 November 2020

\section{References}

1. Siddiqui F, Liu AK, Watkins-Bruner D, et al. Patient-reported outcomes and survivorship in radiation oncology: overcoming the cons. J Clin Oncol. 2014; 32:2920-7.

2. Basch E, Reeve BB, Mitchell SA, et al. Development of the National Cancer Institute's patient-reported outcomes version of the common terminology criteria for adverse events (PRO-CTCAE). J Natl Cancer Inst. 2014;106:dju244.

3. Dueck AC, Mendoza TR, Mitchell SA, et al. Validity and reliability of the U.S. National Cancer Institute's patient-reported outcomes version of the common terminology criteria for adverse events (PRO-CTCAE). JAMA Oncol. 2015;1:1051-9.

4. Hay $\mathrm{J}$, Atkinson TM, Reeve BB, et al. Cognitive interviewing of the U.S. National Cancer Institute's patient-reported outcomes version of the common terminology criteria for adverse events (PRO-CTCAE). Qual Life Res. 2014;23:257-69.

5. Atkinson TM, Ryan SJ, Bennett AV, et al. The association between clinicianbased common terminology criteria for adverse events (CTCAE) and patient-reported outcomes (PRO): a systematic review. Support Care Cancer. 2016;24:3669-76.

6. Arnold B, Mitchell SA, Lent $L$, et al. Linguistic validation of the Spanish version of the National Cancer Institute's patient-reported outcomes version of the common terminology criteria for adverse events (PRO-CTCAE). Support Care Cancer. 2016. https://doi.org/10.1007/s00520-015-3062-5.

7. Kirsch M, Mitchell SA, Dobbels F, et al. Linguistic and content validation of a German-language PRO-CTCAE-based patient-reported outcomes instrument to evaluate the late effect symptom experience after allogeneic hematopoietic stem cell transplantation. Eur J Oncol Nurs. 2015;19:66-74.

8. Baeksted C, Nissen A. Pappot Helle, et al. Danish translation and linguistic validation of the U.S. National Cancer Institute's patient-reported outcomes version of the common terminology criteria for adverse events (PRO-CTCA E). J Pain Symptom Manag. 2016;52:292-7.

9. US NCI PRO-CTCAE. https://healthcaredelivery.cancer.gov/pro-ctcae/ countries.html. Accessed on 30 August 2018.
10. McCarthy N. The world's most spoken languages. Statista, 2018. Retreived from https://www.statista.com/chart/12868/the-worlds-most-spokenlanguages/ Accessed on 30 August 2018.

11. Wild D, Grove A, Martin M, et al. Principles of good practice for the translation and cultural adaptation process for patient-reported outcomes measures: report of the ISPOR task force for translation and cultural adaptation. Value Health. 2005;8:94-104.

12. WHO. Process of translation and adaptation of instruments; 2016. Retrieved from http://www.who.int/research_tools/translation/en/ Accessed on 31 March 2011.

13. Lasch $\mathrm{KE}$, Marquis $\mathrm{P}$, Vigneux $\mathrm{M}$, et al. $\mathrm{PRO}$ development: rigorous qualitative research as the crucial foundation. Qual Life Res. 2010;19:1087-96.

14. Willis GB. Cognitive interviewing. Thousand Oaks: Sage; 2005.

15. Willis $G$. Analysis of the cognitive interview in questionnaire design. Understanding qualitative research. New York: Oxford University Press; 2015

16. Willis $G B$, Reeve BB, Barofsky I. The use of cognitive interviewing techniques in quality of life and patient-reported outcomes measurement. In: Lipscomb J, Gotay CC, Snyder CF, editors. Outcomes assessment in cancer: findings and recommendations of the cancer outcomes measurement working group: Cambridge University Press; 2004. p. 610-22.

17. Singapore Cancer Registry Annual Registry Report 2015. Retrieved from https://www.nrdo.gov.sg/docs/librariesprovider3/Publications-Cancer/ cancer-registry-annual-report-2015_web.pdf?sfvrsn=10 Accessed on 30 August 2018.

18. Wallen GR, Feldman RH, Anliker J. Measuring acculturation among central American women with the use of a brief language scale. J Immigr Health. 2002;4:95-102.

19. Brod M, Tesler LE, Christensen TL. Qualitative research and content validity: developing best practices based on science and experience. Qual Life Res. 2009;18:1263-78.

20. Sireci SG. The construct of content validity. Soc Indic Res. 1998;45:83-117.

21. Cheng KKF, Clark AM. Qualitative methods and patient-reported outcomes: measures development and adaptation. Int J Qual. 2017;16:1-3.

\section{Publisher's Note}

Springer Nature remains neutral with regard to jurisdictional claims in published maps and institutional affiliations.

\section{Ready to submit your research? Choose BMC and benefit from:}

- fast, convenient online submission

- thorough peer review by experienced researchers in your field

- rapid publication on acceptance

- support for research data, including large and complex data types

- gold Open Access which fosters wider collaboration and increased citations

- maximum visibility for your research: over $100 \mathrm{M}$ website views per year

At BMC, research is always in progress.

Learn more biomedcentral.com/submissions 